Canadian Journal of Physics

Canadian

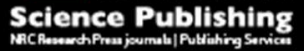

Revue canadienne de physique

\title{
de Broglie Clock, Electron Channeling and Time in Quantum Mechanics
}

\begin{tabular}{|r|l|}
\hline Journal: & Canadian Journal of Physics \\
\hline Manuscript ID & cjp-2017-0571.R2 \\
\hline Manuscript Type: & Article \\
\hline Date Submitted by the Author: & 05-Feb-2018 \\
\hline Keyword: & $\begin{array}{l}\text { electron channeling, time operator, relativistic quantum mechanics, } \\
\text { crystals, solid state }\end{array}$ \\
\hline $\begin{array}{r}\text { Is the invited manuscript for } \\
\text { consideration in a Special } \\
\text { Issue? : }\end{array}$ & N/A \\
\hline \\
\hline
\end{tabular}

\section{SCHOLARONE ${ }^{\text {tw }}$}

Manuscripts 


\title{
de Broglie Clock, Electron Channeling and Time in Quantum Mechanics
}

\author{
M. Bauer \\ Instituto de Física \\ Universidad Nacional Autónoma de México \\ e-mail: bauer@fisica.unam.mx
}

April 26, 2018

\begin{abstract}
De Broglie's association of a wave to particles is a fundamental concept in the quantum mechanical description of nature. The wave oscillation is referred alternatively as the "de Broglie clock", the "Compton clock", or the "de Broglie periodic phenomenon". In the present paper it is shown that Dirac's relativistic quantum mechanics, complemented with the dynamical time operator recently introduced, provides a consistent theoretical description of: a) the generation of the de Broglie wave through Lorentz boosts; b) the characteristics of the resonance observed in electron channeling through thin crystals as responding to both the periodicity derived from the adjustment of the de Broglie period to the crystal interatomic distance (resonance energy) and the periodicity of the predicted trembling motion (Zitterbewegung). One can conclude that the channeling experiments provide the first direct evidence of the electron Zitterbewegung, and that the de Broglie period is an intrinsic property of matter arising from a self adjoint dynamical time operator.
\end{abstract}

\section{Introduction}

It is well known that the daring assumption made by de Broglie of associating a frequency to a particle's mass[1] gave rise to "wave quantum mechanics" as formulated by Schrödinger. The linearity of the proposed differential equation introduced the possibility of wave interference, a phenomenon that was confirmed soon afterwards with electrons[2] and through the years with neutrons, atoms and large molecules, as well with many technological applications in physics, chemistry, biology, industry, medicine, etc. based on the de Broglie relation $\lambda=h / p$ where $h$ is the Planck constant, $p$ the particle momentum and $\lambda$ the associated wave length. Although still subject to interpretative questions, the "wave nature" of matter is now a well accepted element of the description of the physical world. 
Claims of a more direct manifestation of the de Broglie property have been made, supported by an electron channeling experiment, in which relativistic electrons are aligned along a major axial direction of a thin single crystal. A resonance peak inside the expected inhibited transmittivity with respect to other directions occurs at an energy such that electrons pass atom sites with a frequency equal to the de Broglie frequency[3, 4, 5, 6] (to quote: "In other words, observed is a "W" pattern instead of a "U"[3]). Although the predicted resonance energy under this correspondance is close to the observed one, the transmittivity is badly reproduced by a Monte Carlo calculation of a classical trajectory simulated by a series of segments of length equal to the interatomic distance. Surprisingly, a good fit is obtained when segments of half the interatomic distance are assumed, more in accordance with the periodicity of the Zitterbewegung ("trembling motion") phenomenon, noted to begin with by Schrödinger[7]. It is then pointed out that more theoretical and experimental work is required to understand the process.

Dirac's relativistic quantum mechanics (RQM) predicts indeed that a free particle is subject to a "trembling motion" (Zitterbewegung) $[8,9,11]$ associated with the interference of positive and negative energy eigenstates. Zitterbewgung is thus always generated, although it may be transitory. This has been effectively illustrated numerically[12, 13]. As its actual frequency is of the order of $10^{21} \mathrm{~Hz}$ and its amplitude of the order of $10^{-3} \AA$, a direct observation was at the time, and still is, beyond technological capabilities. Dirac[8] recognized its existence but noted that any realistic measurement would be averaging over many oscillations and would not be detected.

Currently however, a Zitterbewegung phenomenon has been observed in systems that simulate Dirac's Hamiltonian dynamics[14, 15]. Here the parameters that substitute for the electron mass and the speed of light give rise to predicted frequencies that are within the range of present technology[16]. Zitterbewegung has been the object of extensive research, most interestingly along the line of providing an explanation of electron's intrinsic magnetic moment and spin, as well as introductory of a non commutative geometry when a minimum spacetime scale of the order of the Compton wavelength is considered[17, 18, 19, 20, 13, 21]. Additional references can be found in the cited papers.

The possible existence of an intrinsic time property is also supported by the introduction of a self-adjoint time operator in Dirac's relativistic quantum mechanics (RQM)[22]. It circumvents the well known Pauli objection[23] by generating energy shifts within either the positive (or negative) energy branch through continuum momentum displacements. It is also shown to generate the Lorentz boost that gives rise to the de Broglie wave, with the corresponding de Broglie period $h / m_{0} c^{2}$ [24] (to quote Baylis: "What in the rest frame is a synchronized oscillation in time is seen in the laboratory to be a wave in space".[25]).Finally, it also provides a description of the tunneling observations in attosecond optical ionization experiments[26]

In the present paper, it is shown that Dirac's relativistic quantum mechanics (RQM), complemented with the dynamical time operator, provides a unified consistent description of the resonance observed in channeling experiments, as 
it associates two frequencies to the electron motion: on the one hand, the de Broglie one corresponding to the periodicity of the full spinor and, on the other, the Zitterbewegung one corresponding to a space periodicity that insures the necessary conditions for a classical description of the channeling process. Secton 2 reviews the definition of the time operator and its interpretation as a generator of a unitary transformation that combines a Lorentz boost and a phase shift[24]. In Section 3, the application to the channeling experiment is developed.

\section{Lorentz boosts and the dynamical time oper- ator in RQM}

A dynamical "time operator" has been introduced in analogy to the Dirac free particle Hamiltonian $H_{D}=c \boldsymbol{\alpha} \cdot \mathbf{p}+\beta m_{0} c^{2}$ where $\alpha_{i}(i=1,2,3)$ and $\beta$ are the $4 \times 4$ Dirac matrices, namely $T=\boldsymbol{\alpha} \cdot \mathbf{r} / \mathbf{c}+\beta \tau_{0}[22]$. This operator is clearly selfadjoint and therefor, by Stone's theorem, can be considered the generator of a unitary transformation, namely:

$$
U_{T}(\varepsilon)=e^{-i \varepsilon T / \hbar}=e^{-i \varepsilon\left\{\boldsymbol{\alpha} \cdot \mathbf{r} / \mathbf{c}+\beta \tau_{0}\right\} / \hbar}
$$

where $\varepsilon$ is real and has the dimensions of energy. For infinitesimal transformations $(\delta \varepsilon<<1)$, the transformed Hamiltonian is:

$$
\hat{U}_{T}(\varepsilon) \simeq e^{-i(\delta \varepsilon)\{\boldsymbol{\alpha} \cdot \hat{\mathbf{r}} / c\} / \hbar} e^{-i(\delta \varepsilon) \beta \tau_{0} / \hbar}=e^{-i(\delta \varepsilon) \beta \tau_{0} / \hbar} e^{-i(\delta \varepsilon)\{\boldsymbol{\alpha} \cdot \hat{\mathbf{r}} / c\} / \hbar}
$$

as $\left[i(\delta \varepsilon)(\boldsymbol{\alpha} . \hat{\mathbf{r}} / c \hbar), i(\delta \varepsilon) \beta \tau_{0} / \hbar\right] \propto(\delta \varepsilon)^{2} \approx 0$ (Glauber proof[27, p.442]). The expectation value of the transformed Hamiltonian is:

$$
\begin{aligned}
\left\langle\Psi\left|\hat{U} \hat{H}_{D} \hat{U}^{\dagger}\right| \Psi\right\rangle & \simeq\left\langle\Phi\left|e^{i(\delta \varepsilon) \boldsymbol{\alpha} \cdot \hat{\mathbf{r}} / c \hbar} \hat{H}_{D}(\mathbf{p}) e^{-i(\delta \varepsilon) \boldsymbol{\alpha} \cdot \hat{\mathbf{r}} / c \hbar}\right| \Phi\right\rangle \\
& \simeq\left\langle\Phi\left|\hat{H}_{D}(\mathbf{p}+\{\delta \varepsilon / c\} \boldsymbol{\alpha})\right| \Phi\right\rangle
\end{aligned}
$$

with

$$
|\Phi\rangle=e^{-i(\delta \varepsilon) \beta \tau_{0} / \hbar}|\Psi\rangle
$$

combining a momentum displacement $\delta \hat{\mathbf{p}}=\{(\delta \varepsilon) / c\} \boldsymbol{\alpha}=\left\{(\delta \varepsilon) / c^{2}\right\}$ c $\boldsymbol{\alpha}$ and a phase shift $\delta \varphi=(\delta \varepsilon) \beta \tau_{0} / \hbar$. Through repeated infinitessimal applications one obtains a Lorentz boost from rest to a momentum $\mathbf{p}$ whose expectation value is

$$
<\mathbf{p}>=\left(\varepsilon / c^{2}\right)\langle c \boldsymbol{\alpha}\rangle=\gamma m_{0} \mathbf{v}_{g p}
$$

where $\gamma=\left\{1-\left(v_{g p} / c\right)^{2}\right\}^{-1 / 2}$ is the Lorentz factor and $\mathbf{v}_{g p}$ the group velocity. It follows that:

$$
h / p=h / \gamma m_{0} v_{g p}=h c^{2} / \gamma m_{0} c^{2} v_{g p}=(h / \varepsilon)\left(c^{2} / v_{g p}\right)=(1 / \nu) v_{p h}
$$

which is precisely the wave length as originally assumed by de Broglie[1], that is, the product of the phase velocity times the period derived from the 
Planck relation $\varepsilon=h \nu$.. The rest frame phase $2 \pi m_{0} c^{2} \tau / h$, where $\tau$ is the proper time, is shifted by an amount

$$
\left.\langle\Delta \varphi\rangle=\left\{\varepsilon \tau_{0} / \hbar\right\}\langle\beta\rangle= \pm \gamma m_{0} c^{2} \tau_{0} / \hbar\right)= \pm 2 \pi \gamma m_{0} c^{2} \tau_{0} / h
$$

as $\left\langle\beta>=m_{0} c^{2} /<H_{D}\right\rangle= \pm m_{0} c^{2} / \varepsilon= \pm 1 / \gamma$, for a positive (negative) energy wave packet that contains both positive and negative energy free particle solutions[9]. In the rest frame, Eq.7 requires $\tau_{0}=h / m_{0} c^{2}$ to yield $\Delta \varphi= \pm 2 \pi$ , resulting in $|\Phi\rangle=|\Psi\rangle$ and mantaining the fundamental indeterminacy of the spinor. This is the de Broglie period $[1,25]$.

In Ref. 21 where the time operator is introduced it was shown that the rate of change of the position with respect to the internal time represented by the time operator, namely $\left[\frac{d r(t)}{d T(t)}\right]$, corresponds to the phase velocity, while the rate of change with respect to the external (laboratory) time $\left[\frac{d r(t)}{d t}\right]$ is the group velocity, ant that their product is equal to $c^{2}$. Thus $v_{p h}>c$, in agreement with the property that de Broglie derives for the wave he associates to a material particle by equating Einstein's $E=m c^{2}$ to Planck's $E=\hbar \nu[1]$.Thus, by introducing the dynamical time operator in Dirac's relativistic quantum mechanics, one now obtains a complete theoretical framework for de Broglie's assumption of a wave associated to matter and his identification of the role of the phase velocity and period.

\section{Zitterbewegung and electron channeling - the de Broglie clock}

The Dirac equation for a free particle yields:

$$
\mathbf{r}(t)=\mathbf{r}_{0}+\left(c^{2} \mathbf{p} / H\right) t+(\hbar / 2 H)\left(e^{2 i H t / \hbar}-1\right) \mathbf{F}
$$

where $\mathbf{F}=c \boldsymbol{\alpha}-c^{2} \mathbf{p} / H[9]$. As expressed by Messiah[10], the wave packet average $<r(t)>$ follows a complicated motion resulting from the addition of a rectilinear uniform motion $\langle r(0)\rangle+\left\langle c^{2} p / H\right\rangle t=\langle r(0)\rangle+v_{g p} t$, where $v_{g p}$ is the group velocity, and a rapid oscillatory motion of amplitude and frequency of the order of $h / 2 m c$ and $2 m c^{2} / h$ respectively. Thus the wave packet oscillates with twice the de Broglie frequency while it advances along the rectilinear trajectory. The oscillation arises when both positive and negative energies are present in the wave packet, bringing in a contribution of the operator $\mathbf{F}[9,11]$. This results from the fact that the time evolution of observables is given by the commutator whereas the Dirac matrices anticommute, and therefore:

$$
\left[H_{D}, \boldsymbol{\alpha}\right]=-\left\{H_{D}, \boldsymbol{\alpha}\right\}+2 H_{D} \boldsymbol{\alpha}=-2 c \mathbf{p}+2 H_{D} \boldsymbol{\alpha}
$$

The average displacement from the linear trajectory at time $t$ is repeated at times $t+\Delta t_{n}$ such that $2 H \Delta t_{n} / \hbar=2 \pi n$ with $n=1,2, \ldots$, or equivalently at intervals $\Delta t=2 \pi \hbar / 2 H=h / 2 H=h / 2 m_{0} c^{2} \gamma$. This interval corresponds to the 
Zitterbewegung laboratory period, (denoted as Z-period) $T_{Z}^{l a b}=\left(1 / \nu_{Z}^{l a b}\right)$, that is equal to half the laboratory de Broglie period (denoted as B-period $T_{B}^{l a b}$ ).

An extremely important consequence of the relativistic invariance of Dirac's equation, relevant to what follows, is that after a $2 \pi$ rotation the space wave function reproduces itself but the spin changes sign. It requires a $4 \pi$ rotation to regain the same spinor $[8,9,11]$. Thus there are two periodicities associated with a relativistic electron motion. On the one hand, one Z-period brings a phase change of $2 \pi$ : the position is repeated but the spinor changes sign. On the other hand, also as a consequence of the Lorentz invariance of the Dirac equation $[9,11]$, to obtain the same spinor one needs a $4 \pi$ rotation, i.e., only after every two Z-periods or, equivalently, one B- period. If the interaction between crystal atoms and the electron is spin dependent, the basis for a resonance phenomenon would then require the interatomic distance $d z$ (atoms aligned in the z-direction) to be equal to $L=2 T_{Z}^{l a b} \mathbf{v}_{g p}=T_{B}^{l a b} \mathbf{v}_{g p}$. This equality defines the group velocity $v_{g p}$ needed and consequently the resonance energy. One has thus provided a formal theoretical justification for the assumption $L=d z$ made in Refs. $(4,5)$ that yields an energy of $80.876 \mathrm{MeV}$, very close to the observed $81.1 \mathrm{MeV}$ in the experiment, as shown in Fig.4 of Ref.3, here reproduced as Fig.1, and derived in Appendix A.

Lindhard[28] demonstrated that under certain conditions classical mechanics can be applied to the channeling case (involving a series of collisions), even though individual scatterings may not be amenable to classical treatment, especially at relativistic energies. This is stated in the review paper of Gemmell[29] that provides an extensive discussion of the basis for the classical theory of channeling. To quote: "Thus it is central to the classical idea of channeling that the encounters of channeled particles with rows or planes be determined by many small-angle scatterings and that the particle do not feel individual atoms. Under these conditions, the continuum model asserts that to a good approximation the motion of channeled particles is determined by a continuum potential, i.e., a potential obtained by replacing the actual periodic potential by a potential averaged over a direction parallel to the row". This theoretical approach, in which the electron trajectory is calculated classically by a Monte Carlo method, was adopted by the team that carried the experiment $[3,4]$. However, the phenomenological Montecarlo calculation does not yield the experimental results when the segments are taken equal to the interatomic distance $d z$, assumed to be attained at B period intervals, as seen also in Fig.1. It is then noted that, surprisingly, the results are reproduced at the same energy (thus same group velocity and same B-period) if the segments for the consecutive classical scatterings are taken to be equal to half the distance $d z$. This is the distance travelled during one Z-period where position is repeated although with a change in sign of the spinor.

As shown above, the resonance energy is such that the scattering centers at $d z$ intervals are found where the full spinor is reproduced. There is no scattering halfway between crystal row atoms. At this energy the portion of the electron beam that undergoes a glancing collision at one atom will continue to experience glancing collisions along the atomic row, reducing or evading the blocking effect 


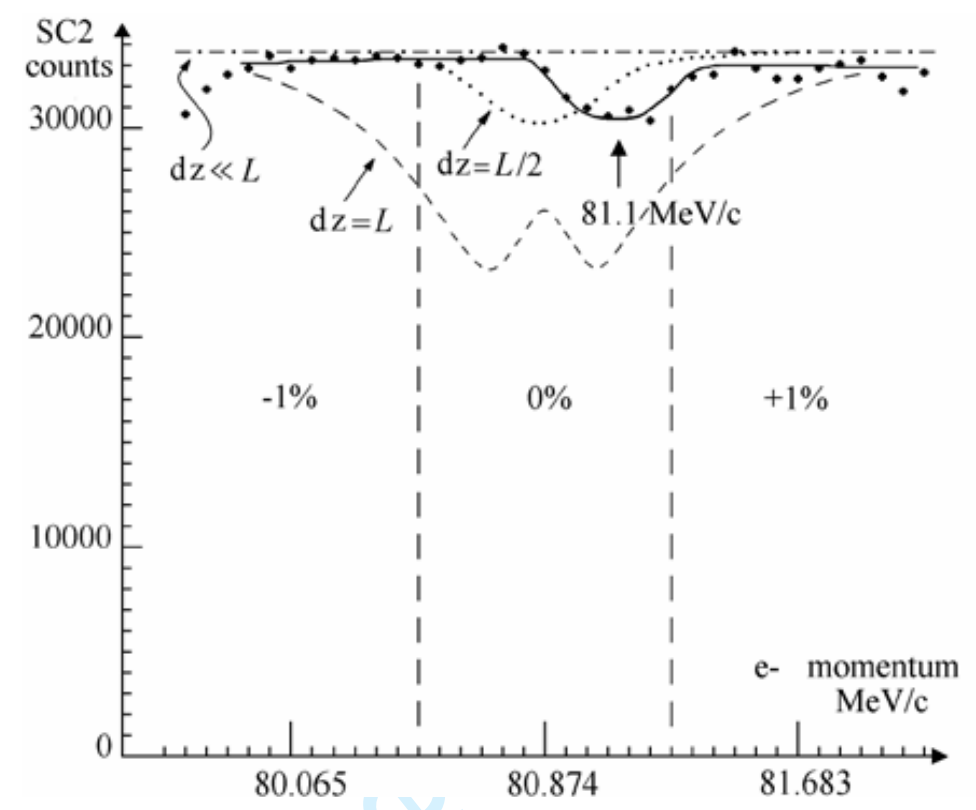

Figure 1: (Fig.4 of Ref.3) Dots: experimental counts vs. electron momentum at $\theta=0^{\circ}$. The dots are corrected data and the full line is to guide the eyes. Dashed curve: result of the model for $d z=L$. Dotted curve: result for $d z=L / 2$. Dashed-dotted curve:result of the model for $d z<<L$.

and thus giving rise to the intermediate peak in the depressed transmission probability. On the other hand, because of Zitterbewegung the spatial part of the spinor is repeated every $d z / 2$ displacement along the linear trajectory and this is taken into account by the successful classical calculation. Appendix B advances a possible explanation on how this change affects the calculation's free parameters to yield the result close to the experimental observation.

In view of this, it is now concluded that the successfull classical calculation, that does not involves spin, is reflecting the space periodicity of Zitterbewegung, whereas the resonance energy is associated with the actual group velocity and the de Broglie periodicity, both present in the time dependence of the free electron motion under the relativistic Dirac Hamiltonian.

The remaining difference between the theoretical and the experimental resonant energies[3], termed compatible with the accuracy $( \pm 0.3 \%)$ of the floating wire calibration, could be accounted by considering that the electron motion in the crystal is represented by the free-particle Hamiltonian with an effective mass $m *$ instead of $m_{0}$ to take into account the average interaction of the electron with the crystal atoms. Following this assumption through the derivation in Appendix A yields $\left(m^{*} / m_{0}\right)^{2}=(81.1) /(80.876)=1.00225$ and $m^{*}=1.0013 m_{0}$. 


\section{Conclusion}

Recently, direct observation of the intrinsic oscillation assumed (the "de Broglie clock") has been brought forward as an experimental possibility. In addition, the trembling motion (Zitterbewegung) of particles satisfying the Dirac relativistic equation of motion has been observed in systems that simulate Dirac's Hamiltonian dynamics. The experimental search for a "de Broglie clock" implies it is considered an observable intinsic property. In the present paper it has been shown that Dirac's relativistic Hamiltonian $H=c \boldsymbol{\alpha} \cdot \mathbf{p}+\beta m_{0} c^{2}$, complemented with a dynamical time operator $T=\boldsymbol{\alpha} \cdot \mathbf{r} / c+\beta h / m_{0} c^{2}$ that generates Lorentz boosts, provides a consistent theoretical support of de Broglie's assumption and consequently a description of:

a) the transformation into a moving electron de Broglie wave of the predicted oscillation in the rest frame - the de Broglie clock, now associated to an internal observable;

b) the fact that the resonance energy observed in electron channeling, that results from equating the de Broglie wave length to the distance $d z$ between atoms, corresponds to the $4 \pi$ rotation periodicity of the spin, as would be required by an interaction between crystal atoms and the electron that includes a spin dependence;

c) the fact that the successful Monte Carlo non spin dependent classical description of the channeling experiment follows the Zitterbewgung $2 \pi$ rotation periodicity of the position evolution.

One can therefore conclude that the channeling experiment provides the first direct evidence of electron's Zitterbewegung, by responding to both the periodicity of the spacial position and the periodicity of the spin. In addition, consistency with the above results requires the undefined parameter $\tau_{0}$ introduced in the dynamical time operator to be equal to $h / m_{0} c^{2}$, the de Broglie period, asserting its role as an intrinsic property of matter. This also supports the de Broglie period as that of an internal clock directly linked to mass, as has been suggested recently[31]. Finally, the existence of a dynamical time operator may be relevant to the formulation of quantum gravity[32].

Acknowledgement. The author wishes to recognize the unknown referees for their thoughtful observations and questions.

\section{References}

[1] L. de Broglie, Ph.D. thesis; Ann. Phys. Ser. 10 ${ }^{\mathrm{e}}$, t. III (1925). English translation in Annales de la.Fondation.Louis de Broglie 17, 92 (1992)

[2] C.J Davisson, and L.H. Germer, Nature 119, 558 (1927)

[3] P. Catillon et al., Found. Phys. 38, 659 (2008)

[4] M. Gouanère et al., Annales de la Fondation Louis de Broglie 30, 109 (2005)

[5] G. Lochak, Annales de la Fondation Louis de Broglie 30, 115 (2005) 
[6] J. Remillieux et al., , Nuc. Instrum. Methods B 355, 193 (2015)

[7] E. Schrödinger, , Berliner Ber., 418 (1930); Berliner Ber, 63 (1931)

[8] P.A.M. Dirac, "The principles of quantum mechanics" (4th ed.), Oxford, Clarendon Press (1958).

[9] B. Thaller, "The Dirac Equation", Springer-Velag, Berlin Heidelberg New York (1992)

[10] Messiah, A., "QUANTUM MECHANICS", Vol. II, 4th ed., p.951, NorthHolland Publishing Company, Amsterdam/John Wiley \& Sons, Inc, New York London Sidney (1966)

[11] W. Greiner, "Relativistic Quantum Mechanics - Wave equations", (3 ${ }^{\mathrm{d}}$ ed.) Springer, Berlin Heidelberg New York (2000)

[12] B. Thaller, "Visualising the kinematics of relativistic wave packets", arXiv:quant-ph/0409079v1 (2008)

[13] S.T. Park, Phys. Rev. A 86, 062105 (2012)

[14] L.J. LeBlanc et al., New J. Phys. 15, 073011 (2013)

[15] R. Gerritsma et al., Nature 463, 68 (2010)

[16] G. Dávid and J. Cserti, Phys. Rev. B 81, 121417(R) (2010)

[17] K. Huang, Am. J. Phys. 20, 479 (1952)

[18] A.O. Barut and A.J. Bracken, Phys. Rev. D 23, 2454 (1981)

[19] D. Hestenes, Found. Phys. 40, 1 (2010)

[20] B.G. Sidharth, Int. J. Theor. Phys. 48, 497 (2009)

[21] S. Sasabe J. Mod. Phys. 5, 534 (2014)

[22] M. Bauer, Int. J. Mod. Phys. A 29, 1450036 (2014)

[23] W. Pauli, "The general principles of quantum mechanics", Springer-Verlag, Berlin Heidelberg (1980), footnote p.63

[24] M. Bauer, Eur. J. Phys. 38, 035402 (2017)

[25] W.E. Baylis, Can. J. Phys. 85, 1317 (2007)

[26] M. Bauer, Phys. Rev. A .022139.(2017)

[27] A. Messiah, "QUANTUM MECHANICS", Vol. I, 4th ed., p.442 NorthHolland Publishing Company, Amsterdam/John Wiley \& Sons, Inc, New York London Sidney (1966) 
[28] J. Lindhard, K. Dan. Vidensk. Selsk, Mat. Fys. Medd. . 34, 1 (1965)

[29] D.S. Gemmell, Rev. Mod. Phys. 46, 129 (1974))

[30] J.U. Andersen, K. Dan. Vidensk. Selsk, Mat. Fys. Medd. 36, No.7 (1967)

[31] S.Y. Lan, et al., Science 339, 554 (2013)

[32] M. Bauer, arXiv:gr-qc/1605.01659 (2016)

\section{Appendix A - Resonance energy in electron channeling}

In a channeling set-up the rectilinear motion would be aligned along the crystal atomic row. Then, as pointed above, the electron wave packet maximum deviation may be made to coincide with crystal atoms given the appropiate group velocity and consequently the appropiate energy. In the experiment cited, the requirement was made that the electron wave packet should advance the interatomic distance $d z$ in one B period, i.e., $L=v_{g p} T_{B}^{l a b}=d z$. Now $T_{B}^{l a b}=$ $\left(h \gamma / m_{0} c^{2}\right)$ since the de Broglie linear frequency in the laboratory is $\nu_{B}^{l a b}=$ $\left(m_{0} c^{2} / h \gamma\right)$. Then:

$$
v_{g p}=\left(d z / T_{B}^{l a b}\right)=(d z)\left(m_{0} c^{2} / h \gamma\right)
$$

Defining $d z\left(m_{0} c^{2} / h c\right)=a$ and $\left(v_{g p} / c\right)=b$, it follows that:

$$
(b \gamma)^{2}=b^{2}\left(1-b^{2}\right)^{-1}=a^{2},
$$

and finally

$$
b=\left(v_{g p} / c\right)=a /\left(a^{2}+1\right)^{1 / 2} \approx 1-1 /\left(2 a^{2}\right) \text { if } a>>1
$$

In the reported experiment $d z=3.84 \AA=3.84\left(10^{5}\right) F$. Then

$$
a=d z\left(m_{0} c^{2}\right) / h c=(1 / 2 \pi) d z\left(m_{0} c^{2}\right) / \hbar c=158.2707
$$

and the motion is seen to be highly relativistic. The laboratory energy is:

$$
\begin{aligned}
E & =m_{0} c^{2} \gamma \simeq m_{0} c^{2} a=m_{0} c^{2} d z\left(m_{0} c^{2} / h c\right)= \\
& =0.511(158.2707) \mathrm{MeV}=80.876 \mathrm{MeV}
\end{aligned}
$$

On the other hand, from the above derivation, if the full interatomic distance $d z$ is to be attained after one $\mathrm{Z}$ period, or equivalently $2 d z$ in a $\mathrm{B}$ period, the energy must be $E=161.752 \mathrm{MeV}$. The corresponding transmitivity would be as given by the curve $L=d z$ in Fig.1. Finally, a resonance energy $E=$ $40.438 \mathrm{MeV}$ follows from requiring the distance $d z$ to be travelled in two B periods or four $\mathrm{Z}$ periods. All these situations are recognized in Refs.(18,19), suggesting that the phenomenon should also be observable at these energies.

Appendix B - On the Montecarlo calculation[3, 4, 5]

The calculation in Refs. 3,4 and 5 has as independent parameters the intensity $K$ of confining potential $U\left(x_{i}, y_{i}, z_{i}\right)=K x_{i}^{2} y_{i}^{2} z_{i}^{2}$, and the range $\sigma$ of the 
Gaussian shape used to introduce a fluctuating length at each step. Taking the de Broglie wave length equal to the interatomic distance, $L=d z$, the deviation angle at each step in the $\mathrm{x}$-direction of the transverse plane is given as:

$$
d \alpha_{i}=2 L K y_{i}^{2} z_{i}^{2} x_{i} / m=2 d z K y_{i}^{2} z_{i}^{2} x_{i} / m
$$

and similarly in the $y$-direction (see Eqs.(2) and (3) of Ref.4. If one considers now $L=2 d z$ one can rewrite this equation as

$$
d \alpha_{i}=2 d z K^{\prime} y_{i}^{2} z_{i}^{2} x_{i} / m
$$

with $K^{\prime}=2 K$. This is equivalent to conserve $L=d z$, and consequently the group velocity and energy, but to double the strength of the confining potential and thus the restoring force. This results in a contraction of all dimensions. As such, it can be expected to reduce the width of the transmission dip. In addition the normalized Gaussian was given the value $\sigma=L / 4=d z / 4$. If now $L=2 d z$, the range parameter becomes $\sigma^{\prime}=d z / 2$, increasing its range and reducing the maximum value by $1 / 2$. As pointed out in Ref.4, "the parameter $\sigma$ acts only on the dip amplitude, not on its width". Using a more extended Gaussian function will reduce the amplitude of the transmission dip. Thus the above changes in $K$ and $\sigma$ are expected to reduce both the width and the amplitude of the transmission dip, bringing the calculation in closer agreement with the experiment, as occurred in Ref.3. 